\title{
A Note on the Barotropic Instability of the Tropical Easterly Current*
}

\author{
By Tsuyoshi Nitta and M. Yanai \\ Geophysical Institute, Tokyo University, Tokyo \\ (Manuscript received 13 February 1969, in revised form 24 April 1969)
}

\section{Introduction}

The stability of barotropic zonal currents in the atmosphere with respect to wave disturbances has been studied by Kuo (1949), Lipps $(1962,1965)$ and others. But these studies were mostly concerned with the case of westerly zonal currents. The barotropic stability of easterly zonal currents has not been fully examined.

It has been known that there exist synopticscale wave disturbances in the trade easterlies in the Pacific and in the Caribbean Sea (Palmer, 1951; Riehl, 1954). These wave disturbances are known to possess the "cold-core" structure. The air to the east of the wave axis, where the most active weather is observed, is colder than the surrounding air. The wave accompanies upward motions to the east of the wave axis and downward motions to the west (Krishnamurti and Baumhefner, 1966; Yanai and Nitta, 1967). The mechanism that maintains this cold-core circulation is still somewhat a puzzle. Palmer (1951) and Yanai (1961) have suggested that kinetic energy of the wave is supplied from the trade easterly current which has a horizontal shear. Charney (1963) argues that in the absence of condensation large-scale motions in the tropics tend to be quasi-horizontal and quasi-nondivergent. If this argument is correct, the barotropic instability may be of some importance in the dynamics of tropical disturbances.

2. Difference between the instability properties of the westerly current and those of the easterly current

For simplicity we discuss the stability of a barotropic zonal current confined between two latitudes based on the linearized vorticity equation

* Division of Meteorology, Contribution No. 171.

$$
\left(\frac{\partial}{\partial t}+U \frac{\partial}{\partial x}\right) \nabla^{2} \phi+\left(\beta-U^{\prime \prime}\right) \frac{\partial \phi}{\partial x}=0
$$

where $x$ and $y$ are Cartesian co-ordinates directed toward the east and north respectively, $\phi$ the stream function for the perturbed motion, $\beta=d f / d y, f$ the Coriolis parameter, $U(y)$ is the basic current and a prime denotes a differentiation with respect to $y$. We set

$$
\phi(x, y, t)=\phi(y) e^{i \alpha(x-c t)},
$$

then (1) becomes

$$
(U-c)\left(\phi^{\prime \prime}-\alpha^{2} \phi\right)+\left(\beta-U^{\prime \prime}\right) \phi=0
$$

where $\alpha$ is the wave number and $c$ the phase velocity of the disturbance. $c$ may be complex in general, i.e.,

$$
c=c_{r}+i c_{i}
$$

We impose boundary conditions at two latitudes $y=0$ and $y=2 b$ as

$$
\phi(0)=\phi(2 b)=0
$$

We divide the interval $2 b$ into 20 subdivisions and obtain $c$ and $\phi$ by the method of finite differences. The validity of the finite difference method and its accuracy were discussed at length in a previous paper (Yanai and Nitta, 1968). In this study we use $\beta=2.29 \times 10^{-11} \mathrm{~m}^{-1} \mathrm{sec}^{-1}$ (on the equator).

To demonstrate the difference between the stability characteristics of the westerly basic current and those of the easterly current, we consider simple velocity profiles given by

$$
\begin{aligned}
& U_{W}=U_{\max } \sin ^{2}\left(\frac{\pi y}{2 b}\right) \\
& U_{E}=-U_{\max } \sin ^{2}\left(\frac{\pi y}{2 b}\right)
\end{aligned}
$$

where $U_{W}$ and $U_{E}$ denote westerly and easterly zonal current respectively, and $U_{\max }(>0)$ is the maximum zonal wind speed. These profiles have inflection points at $y=y_{t}=b / 2$. The minimum 
critical value of $U_{\max }$ denoted by $U_{c}$ for which the gradient of absolute vorticity $\beta-U^{n}$ vanishes somewhere in the belt is given, in both cases, by $U_{c}=2 \beta b^{2} / \pi^{2}$. When $U_{\max }$ is greater than $U_{c}$, $\beta-U^{\prime \prime}=0$ is satisfied at $y=y_{k}$ in the interval $0<y<b$, and unstable waves may exist.

In the case of the westerly basic current that has a symmetric velocity profile, Kuo (1949) proves that neutral waves whose phase velocity is greater than the maximum zonal current velocity do not exist. On the other hand neutral waves whose phase velocity is less than the minimum zonal current velocity can exist. Kuo further found that disturbances are unstable in the range between two critical wavelengths. The lower critical wavelength denoted by $L_{\kappa}$ is the characteristic wavelength of the neutral wave which has a phase velocity $c=U\left(y_{k}\right)$. The upper critical wavelength, $L_{0}$ is the minimum wavelength of

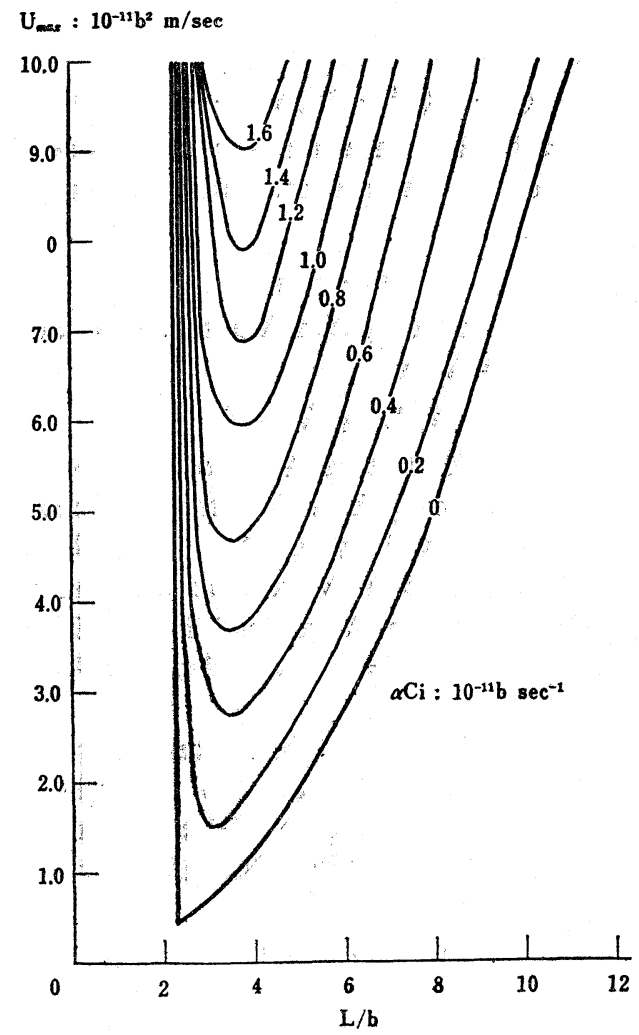

Fif. 1. The critical curve and the growth rate of the unstable wave in the westerly basic flow given by (5). The growth rate is given in units of $10^{-11} b \mathrm{sec}^{-1}$. The numerical value of $b$ (the half width of basic flow) should be given in meters. the Rossby-Haurwitz wave which has a phase velocity $c=U_{\text {min }}$.

We can show that there is only one mode of unstable wave for both the velocity profiles (5) and (6) and that their lower critical wavelengths are identical, i.e.,

$$
L_{k}=\frac{4 b}{\sqrt{3}}=2.31 b
$$

On the other hand, we can show that in the case of easterlies given by (6) the upper critical wavelength does not exist. In other words, there are no long neutral waves of Rossby and Haurwitz and all disturbances are unstable for wavelengths longer than $L_{\kappa}$, providing $U_{\max }>U_{c}$.

The difference between the stability characteristics of the westerly current and those of the easterly current is examined by numerical calculations of the growth rate and the critical curves for instability by the method of finite differences. Figs. 1 and 2 show the curves of the growth rate

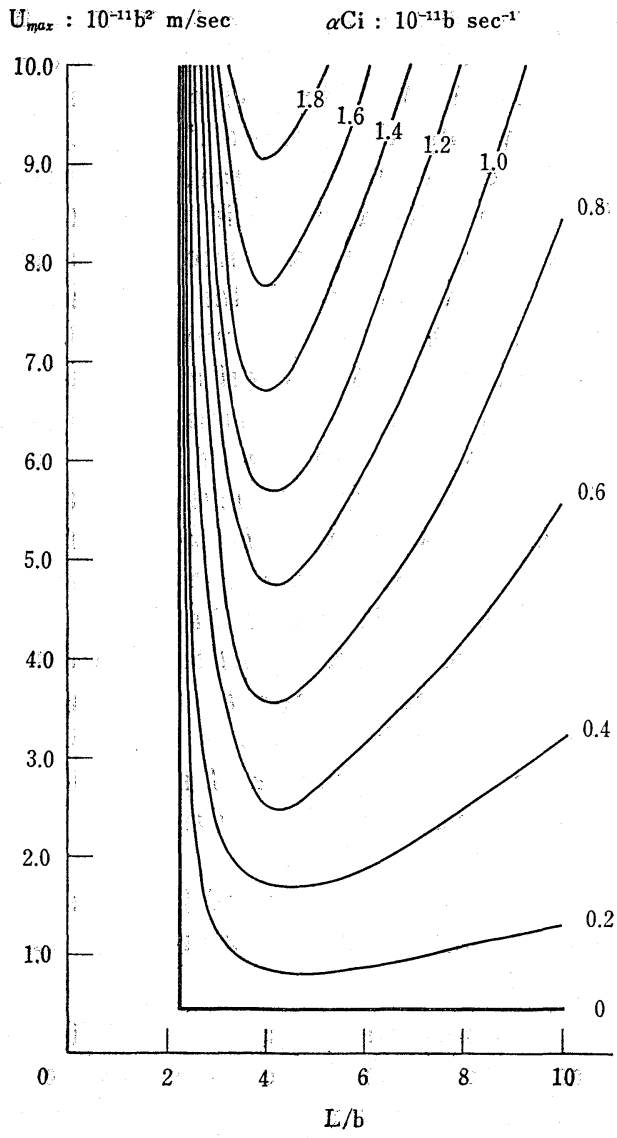

Fig. 2. The critical curve and the growth rate of the unstable wave in the easterly basic flow given by (6). 
of amplified disturbances for the westerly and the easterly velocity profiles respectively, For the westerly zonal current, unstable waves are confined between two critical wavelengths. On the other hand, all disturbances which have wavelengths longer than $2.31 b$ are unstable for the easterly current. The growth rate of amplified disturbances in the easterlies is larger than that in the westerlies. For a moderate horizontal shear of the zonal current, the most preferred scale of unstable waves in the easterlies is larger than that in the westerlies. We remark that the difference of the stability characteristics between the westerly and the easterly currents arises from the $\beta$-term in (2). If we choose the $x$-coordinate directed westward, the form of (2) would be unchanged except for the sign of the $\beta$-term. An analogous situation exists in the problem of lee waves produced by obstacle which was discussed by Kasahara (1966).

\section{Possibility of instability in the trade easterlies}

We next examine the possibility of barotropic instability in the actual zonal currents in the tropics. Fig. 3a shows latitudinal distributions of the average zonal velocity and absolute vorticity at the surface, 1000-, 850-, 700-, and 600-mb levels observed over the Marshall Islands for June 1958. The velocity distributions which resemble half-jets between the equator and $20^{\circ} \mathrm{N}$ are typical for the western Pacific region. At the surface and at the $1000-\mathrm{mb}$ level, the gradient of absolute vorticity changes its sign at about $8^{\circ} \mathrm{N}$ and amplified disturbances are expected to occur at these levels. Fig. $3 \mathrm{~b}$ shows distributions of the average zonal velocity and absolute vorticity observed over the same area for July 1958. The condition $\beta-U^{\prime \prime}=0$ is met at the 700 - and $600-\mathrm{mb}$ levels. Fig, 4 shows distributions of the monthly mean zonal velocity and absolute vorticity at the $3-\mathrm{km}$ level over the Line Islands area in the eastern Pacific from April to July 1962. The velocity profiles are nearly symmetric with respect to the jet axis which lies on the equator. It is seen that $\beta-U^{\prime \prime}$ is positive everywhere except for the velocity profile observed in July.

Using the Marshall Islands data at the surface of June 1958, we made the computation of the growth rate of amplified disturbances by the finite difference method. In this computation, the position of the boundaries is somewhat arbitrary. For an untisymmetric velocity profile similar to

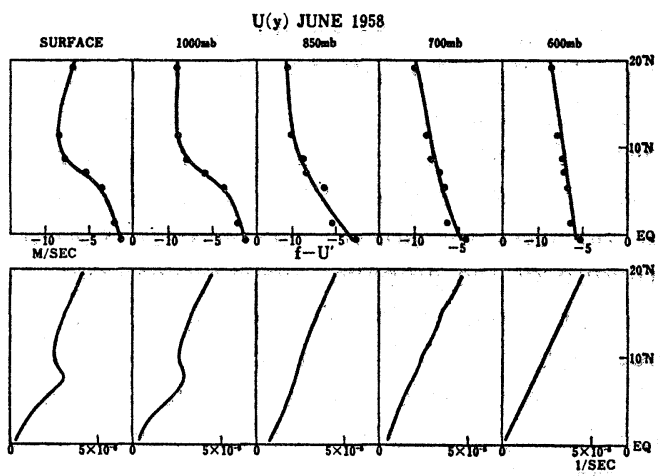

(a)

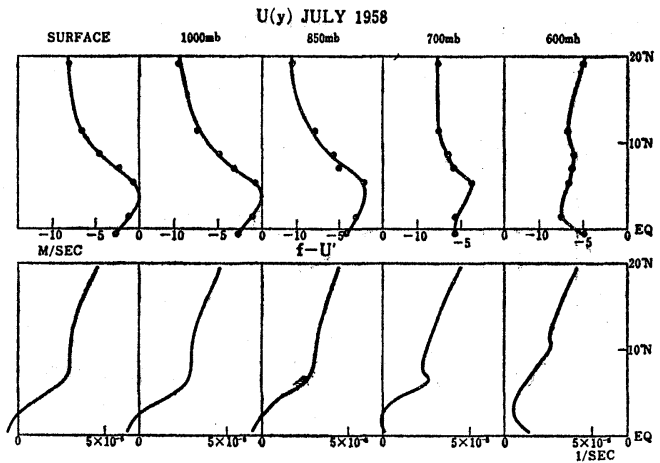

(b)

Fig. 3. The mean zonal current and the corresponding absolute vorticity observed over the Marshall Islands at yarious levels for June 1958 (a) and those for July 1958 (b).

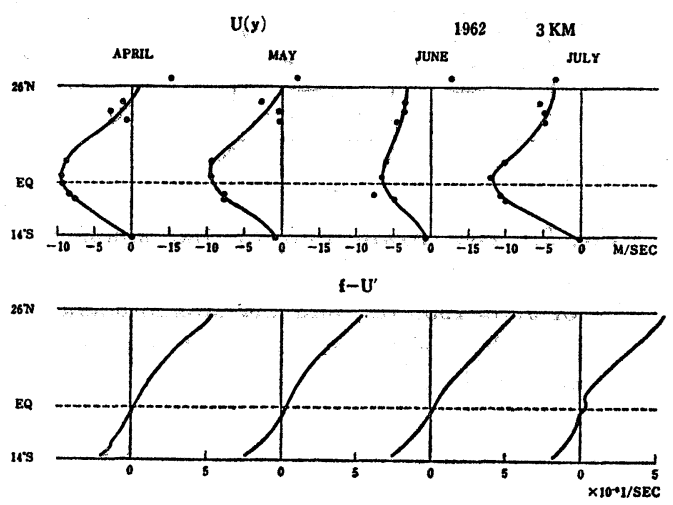

Fig. 4. The mean zonal current and the corresponding absolute vorticity observed at the $3-\mathrm{km}$ level over the Line Islands area from April to July 1962.

the observed profile, the authors examined the dependence of the stability characteristics on the position of boundaries (Yanai and Nitta, 1968). Based on the result of that study and also on an 
empirical fact that the amplitude of wave disturbances in the Marshall Islands area attains its maximum at about $10^{\circ} \mathrm{N}$ and decays off both polewards and equatorwards, we set the boundaries at the equator and at the latitude $20^{\circ} \mathrm{N}$. Figs. $5 \mathrm{a}$ and $5 \mathrm{~b}$ show the growth rate and the horizontal structure of the amplified wave respectively. The most preferred scale of the amplified wave is about $2,000 \mathrm{~km}$ and the maximum growth rate is $2.1 \times 10^{-6} \mathrm{sec}^{-1}$ which corresponds to an e-folding time 5.2 days. The axis of the amplified wave is directed from southwest to northeast. These characteristics of amplified waves are somewhat similar to those of the easterly wave observed in

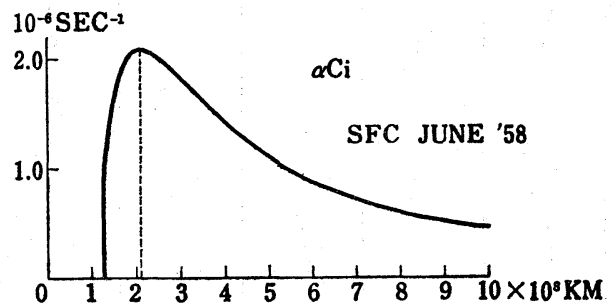

(a)

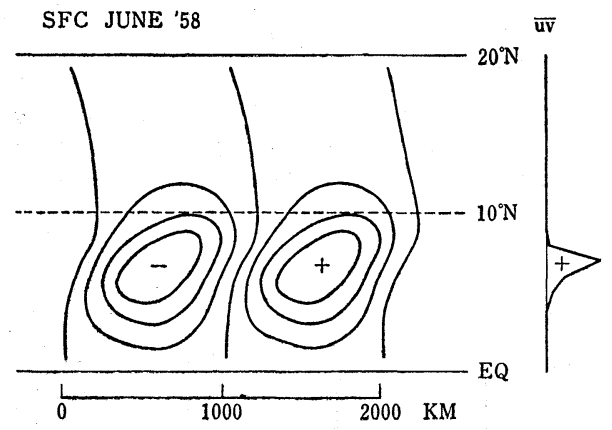

(b)

Fig. 5. The growth rate of the amplified disturbance for the surface velocity profile of June 1958 (a), and the streamfunction and the $u-v$ correlation of the most preferred wave (b). the western Pacific. If the daily velocity profiles were used instead of the monthly mean velocity profile, the growth rate could be larger.

\section{Acknowledgments}

This study was partially supported through a grant from the Japan Society for the Promotion of Science as part of the Japan-U.S. Cooperative Science Program. The authors thank Mrs. M. Ishiguro and Miss I. Tamura for typing the manuscript.

\section{References}

Charney, J.G., 1963: A note on large-scale motions in the tropics. J. atmos. Sci., 20, 607-609.

Kasahara, A., 1966: The dynamical influence of orography on the large-scale motion of the atmosphere. J. atmos. Sci., 23, 259-271.

Krishnamurti, T.N., and D. Baumhefner, 1966: Structure of a tropical disturbance based on solutions of a multilevel baroclinic model. J. appl. Meteor., 5, 396-406.

Kuo, H.L., 1949: Dynamic instability of twodimensional nondivergent flow in a barotropic atmosphere. J. Meteor., 6, 105-122.

Lipps, F.K., 1962: The barotropic stability of the mean winds in the atmosphere. J. Fluid Mech., 12, 397-407.

- 1965: The stability of an asymmetric zonal current in the atmosphere. J. Fluid Mech., 21, 225-239.

Palmer, C.E., 1951: Tropical meteorology. Compendium of Meteorology, Amer. Meteor. Soc., 859-880.

Riehl, H., 1954: Tropical Meteorology. McGraw-Hill Book Company, 392pp.

Yanai, M., 1961: Dynamical aspects of typhoon formation. J. meteor. Soc. Japan, 39, 282-309.

, and Tsuyoshi Nitta, 1967: Computation of vertical motion and vorticity budget in a Caribbean easterly wave. J. meteor. Soc. Japan, 45, 444-466.

approximations for the barotropic instability problem. J. meteor. Soc. Japan, 46, 389-403.

熱帯偏東風の順圧不安定について

新田就 - 柳 井迪 雄 


\section{CORRIGENDA}

"Numerical Simulation of the Blocking Process" By Yukio Kikuchi, in the February 1969 issue of the Journal of the Meteorological Society of Japan (Ser. II, Vol. 47, No. 1) should be corrected as follows.

\begin{tabular}{|c|c|c|c|c|}
\hline Page & Column & line & Wrong & Right \\
\hline 30 & left & 10 from the bottom & $+\nabla(f \nabla x=F)$ & $+\nabla(f \nabla x)=F$ \\
\hline 30 & left & second from the bottom & $\nabla^{2} \phi=\nabla \cdot(f \nabla \phi)$ & $\nabla^{2} \Phi=\nabla \cdot(f \nabla \psi)$ \\
\hline 30 & right & 6 from the top & $i-\frac{\sigma}{a \cos \phi \delta \lambda}$ & $i \frac{\sigma}{a \cos \phi \partial \lambda}$ \\
\hline 31 & left & third from the top & $\times$ & $X$ \\
\hline 32 & right & 6 from the bottom & $T^{\prime} s$ & $T_{s}^{\prime}$ \\
\hline 32 & right & first from the bottom & $a_{1} T^{\prime} s$ & $a_{1} T_{s}^{\prime}$ \\
\hline 33 & left & first from the top & $b_{1} T^{\prime} s$ & $b_{1} T_{s}^{\prime}$ \\
\hline 33 & right & 19 from the bottom & $C=16.667 \mathrm{ly} \mathrm{day}^{-1} \mathrm{~K}^{-1}$ & $c=16.667$ ly day ${ }^{-1} \mathrm{~K}^{-1}$ \\
\hline 33 & right & 10 from the bottom & $+\left(b^{2}+c_{2}-a_{2}\right) T^{\prime}$ & $+\left(b_{2}+c_{2}-a_{2}\right) T^{\prime}$ \\
\hline 33 & right & 9 from the bottom & $T_{s}$ & $T_{s}^{\prime}$ \\
\hline 33 & right & 7 from the bottom & $T_{s}$ & $T_{s}^{\prime}$ \\
\hline 33 & right & 5 from the bottom & $T_{s}$ & $T_{s}^{\prime}$ \\
\hline 34 & left & second from the bottom & Eq. (37) Q & Eq.(37), $Q$ \\
\hline 35 & left & fourth from the top & $+\left(b_{0}+c_{0}+a_{0}\right)$ & $+\left(b_{0}+c_{\nu}-a_{0}\right)$ \\
\hline 35 & right & 15 from the top & $J\left(\psi_{3} \nabla^{2} \psi_{3}+f\right)$ & $J\left(\phi_{3}, \nabla^{2} \phi_{3}+f\right)$ \\
\hline 36 & left & first from the top & $P_{n}^{-m}$ & $P_{n}^{-m}$ \\
\hline 36 & left & 14 from the bottom & $\frac{d \Psi_{\alpha}}{d t}-$ & $\frac{d \Psi_{\alpha}}{d t}=$ \\
\hline 36 & left & 7 from the bottom & $-\frac{\rho_{2}}{R} \sigma D a X a$ & $-\frac{p_{2}}{R} \sigma D_{a} X_{a}$ \\
\hline 36 & right & third from the top & $\int_{-\pi / 2}^{\pi / 2} p_{\alpha}\left(m_{\beta} p_{\beta} \frac{d \rho_{\gamma}}{d \phi}\right.$ & $\int_{-\pi / 2}^{\pi / 2} P_{\alpha}\left(m_{\beta} P_{\beta} \frac{d P_{\gamma}}{d \phi}\right.$ \\
\hline 36 & right & fourth from the top & $\left.-m_{\gamma} p_{\gamma} \frac{d p_{\beta}}{d \phi}\right) d \phi$ & $\left.-m_{\gamma} P_{\gamma} \frac{d P_{\beta}}{d \phi}\right) d \phi$ \\
\hline 36 & right & 9 from the top & $\int_{-\pi / 2}^{\pi / 2} p_{a} p_{b} p_{c} \cos \phi d \phi$ & $\int_{-\pi / 2}^{\pi / 2} P_{a} P_{b} P_{c} \cos \phi d \phi$ \\
\hline 36 & right & 9 from the bottom & $T_{\circ}^{\circ}$ & $T_{0}^{0}$ \\
\hline 42 & left & Fig. 17 & Burdecki 1955) & Burdecki (1955) \\
\hline 42 & right & 13 from the bottom & $\frac{\partial T^{2}}{\partial t}$ & $\frac{\partial \bar{T}_{2}}{\partial t}$ \\
\hline 42 & right & 13 from the bottom & $\frac{p_{2}}{R} \sigma \overline{\omega^{2}}$ & $\frac{p_{2}}{R} \sigma \overline{\omega_{2}}$ \\
\hline 44 & left & 13 from the bottom & $-\gamma_{m} \psi_{3} \frac{\partial h}{\partial \lambda}$ & $-\gamma_{m} \overline{\psi_{3} \frac{\partial h}{\partial \lambda}}$ \\
\hline 45 & left & 23 from the bottom & Symbols, & Symbols. \\
\hline 46 & left & Fig. 23 & 20 day & 30-day \\
\hline 46 & right & 14 from the bottom & $\nabla^{2} \phi_{l}$ & $\nabla^{2} \Phi_{l}$ \\
\hline 47 & left & fourth from the bottom & $1 / \Delta p \omega_{2} \Phi_{2}$ and $g / \Delta p \psi_{2} \tau_{2}$ & $1 / \Delta p \overline{\overline{\omega_{2} \Phi_{2}}}$ and $g / \Delta p \overline{{\overline{\psi_{2}}}_{2} \tau_{2}}$ \\
\hline 49 & & Caption of Table 1 & component & components \\
\hline 49 & & , & Oort 1964) & Oort (1964) \\
\hline 49 & right & 20 from the bottom & at wave number 5). & around this wave number). \\
\hline 49 & right & 8 from the bottom & figure the & figure, the \\
\hline 50 & right & first from the top & From part (1) & From Part (I) \\
\hline 52 & left & 17 from the bottom & $\begin{array}{l}\text { troposphere ocean } \\
\text { continent. }\end{array}$ & $\begin{array}{l}\text { troposphere-Ocean-Continent } \\
\text { system. }\end{array}$ \\
\hline 53 & & 和文アブストラクト & 菊地 & 菊池 \\
\hline 53 & & 下から 5 行目 & 比較した結果 & 比較した. その結果 \\
\hline 53 & & 下から 1 行目 & 角運動輸送 & 角運動量輸送 \\
\hline
\end{tabular}

